\title{
Gradhiva
}

\section{Annette Becker et Octave Debary (dir.). Montrer les violences extrêmes}

Grane, Créaphis Éditions, 2012.

\section{Anne-Solène Rolland}

\section{(2) OpenEdition}

\section{Journals}

Édition électronique

URL : http://journals.openedition.org/gradhiva/2764

DOI : 10.4000/gradhiva.2764

ISSN : 1760-849X

\section{Éditeur}

Musée du quai Branly Jacques Chirac

Édition imprimée

Date de publication : 1 décembre 2013

Pagination : 256-257

ISBN : 978-2-35744-072-2

ISSN : 0764-8928

\section{Référence électronique}

Anne-Solène Rolland, « Annette Becker et Octave Debary (dir.). Montrer les violences extrêmes 》,

Gradhiva [En ligne], 18 | 2013, mis en ligne le 27 novembre 2013, consulté le 22 septembre 2020. URL : http://journals.openedition.org/gradhiva/2764 ; DOI : https://doi.org/10.4000/gradhiva.2764

Ce document a été généré automatiquement le 22 septembre 2020.

(c) musée du quai Branly 


\section{Annette Becker et Octave Debary (dir.). Montrer les violences extrêmes}

Grane, Créaphis Éditions, 2012.

Anne-Solène Rolland

\section{RÉFÉRENCE}

Annette Becker et Octave Debary (dir.). Montrer les violences extrêmes. Grane, Créaphis Éditions, 2012.

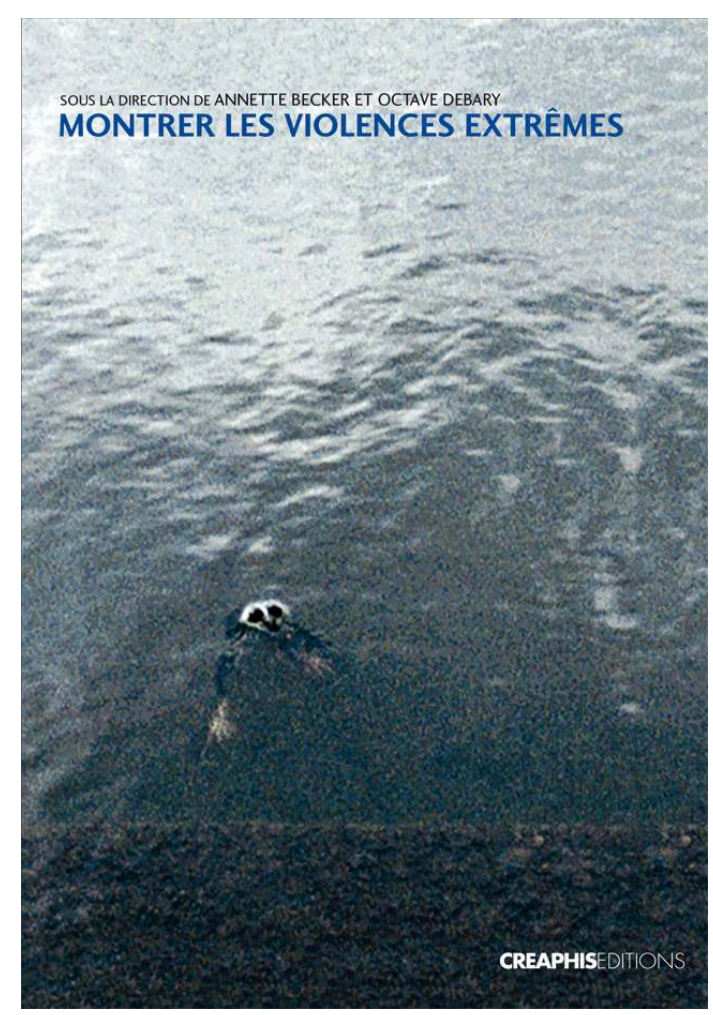


1 En 2013, le festival de Cannes a primé un film du réalisateur franco-cambodgien Rithy Panh au titre évocateur, L'Image manquante. Panh, déjà auteur de plusieurs documentaires sur les crimes des Khmers rouges, a choisi de remplacer cette «image manquante » du massacre de sa famille par des figurines de terre cuite pour dire, malgré l'absence de trace, l'indicible et en garder la mémoire. C'est à cette question toujours actuelle de "comment montrer l'inmontrable» que s'intéresse l'ouvrage dirigé par Annette Becker et Octave Debary, qui fait suite à un colloque organisé par l'Institut national d'histoire de l'art (INHA) et le musée du quai Branly en 2008.

2 Sous la direction d'une historienne des cultures de guerre ${ }^{1}$ et d'un anthropologue spécialiste de la mémoire ${ }^{2}$, l'ambition de cet ouvrage est de "questionner le difficile travail d'historicisation des drames» (p. 7) en plaçant au cœur de la réflexion les disciplines dont la vocation même est de montrer: les musées, les films, les œuvres d'art, les lieux de mémoire. C'est donc sous un angle paradoxal, et parfois provocateur dès l'introduction est mise en cause la capacité des musées à montrer les violences extrêmes (p. 5) -, que l'ouvrage aborde un domaine qui a trait non seulement à l'histoire et à la mémoire mais aussi à la dimension humaine, car profondément émotionnelle, de l'expérience culturelle. Si, évidemment, la Shoah est le cas de "violence extrême» le plus évoqué, d'autres catastrophes sont également abordées, jusqu'au cas contemporain de la mise en musée du 11 Septembre.

3 Les qualités de cet ouvrage sont multiples. D'abord, sa pluridisciplinarité : historiens, philosophes, muséologues, artistes et scénographes sont réunis pour confronter leurs points de vue dans quatre chapitres, "Théoriser", "Créer", "Historiciser ", "Muséographier». Ensuite, le parti de mêler approches théoriques et études de cas offre un aperçu très complet des questions posées. Enfin, en tout cas dans son volet muséologique, il élargit son champ d'études au-delà des musées de guerre et inclut dans son analyse des musées d'histoire des questionnements muséographiques portant sur l'objet, la dimension artistique, la mise en espace et la place du visiteur.

4 Dès l'introduction, sous les auspices de l'œuvre de Paul Ricœur ${ }^{3}$, une affirmation provocatrice est posée: "Les musées sont (aussi) faits pour oublier» (p. 9). Cette analyse de l'oubli, dans sa dimension problématique ou salutaire, sous-tend une partie des articles. Il s'agit de montrer combien la question des catastrophes est complexe, et le besoin d'oubli plus pressant encore que dans toute autre mémoire. L'article de Philippe Braunstein sur les monuments aux morts pose cette question en montrant les choix faits, après chaque conflit, de commémoration de certains aspects et d'oubli d'autres moins consensuels et donc moins propices à créer une mémoire collective fédératrice. L'exemple du mémorial de la guerre du Vietnam à Washington et des polémiques qu'il suscita est à cet égard particulièrement intéressant (p. 188-189).

5 Par cette question de l'oubli délibéré, c'est aussi la part idéologique et politique de la mémoire qui est évoquée à plusieurs reprises : comme le souligne Nina Gorgus, «le thème de la guerre dans les musées reste problématique car un trop grand nombre d'intérêts idéologiques y est toujours associé ${ }^{4} »$ (p. 213). L'exemple récent de la polémique suscitée par l'exposition Foyer fantôme, au Jeu de Paume à l'été $2013^{5}$, présentant des œuvres de la photographe palestinienne Ahlam Shibli, vient étayer les analyses de François Soulages sur la photographie, selon qui «la photographie de guerre va, volontairement ou non, participer au culte idéologique des héros et de leurs commanditaires » (p. 45). 
6 Aborder la mémoire à travers l'oubli, c'est aussi interroger différemment le rapport entre la mémoire et l'histoire: la monstration de l'horreur tendrait «plus vers la commémoration muette que vers la compréhension historique» (p. 10). Les musées comme les lieux de mémoire, par une approche souvent émotionnelle, favoriseraient une mémoire silencieuse, qui tend vers l'oubli, plutôt qu'une explicitation douloureuse qui favorise la mémoire. Nombre d'exemples interrogent cette évocation sensible, comme l'architecture du Jüdisches Museum ${ }^{6}$ de Berlin évoquée par Sophie Wahnich ou le parcours de l'Holocaust Memorial de Washington analysé par Philippe Mesnard, exemple sans doute le plus frappant d'une appropriation mémorielle et d'une abolition de la distance entre le visiteur et le sujet traité.

Cette notion de distance, en particulier de son absence, est un autre concept central de l'ouvrage: distance temporelle avec le sujet abordé, distance entre le visiteurspectateur et son objet, distance émotionnelle envers le sujet traité. Analyser la distance qui sépare le visiteur du propos, c'est rejoindre le débat entre mémoire et histoire du point de vue de l'expérience de visite et analyser la part d'historicité du propos muséographique. Cela permet à plusieurs auteurs d'interroger la place de l'art dans l'évocation des catastrophes. Cette part importante donnée à l'art, y compris à la parole des artistes à laquelle est consacré le chapitre "Créer » (Jochen Gerz, Natacha Nisic, Liza Nguyen et Claire Angelini), est une des originalités de l'ouvrage : au-delà des interrogations propres aux professionnels de l'histoire, cette parole apporte un éclairage nouveau à la réflexion. Le bref récit par Nisic de la réalisation de la photographie, prise à Birkenau, qui illustre la couverture de l'ouvrage (Effroi, 2005) est un témoignage saisissant.

8 La place de l'art dans les musées d'histoire est le thème de trois des articles du premier chapitre: Soulages analyse la place particulière et souvent problématique de la photographie, tandis que l'article de Wahnich, consacré à l'art dans les musées de guerre, et celui de Mesnard, qui aborde la question du "pathos » dans les musées et mémoriaux, se répondent. Sur la base d'études de cas, tous deux interrogent l'équilibre entre art et histoire dans l'évocation de la violence, et la « juste distance » à laquelle tenir le visiteur. Là où Wahnich retient, dans le cas du Jüdisches Museum ou de l'Historial de Péronne, la justification d'une œuvre d'art si elle « a le pouvoir d'abolir l'inactualité douloureuse de l'événement» (p. 29) et "ne vise pas toujours à l'identification mais fabrique un autre régime de temporalité pour historiciser face au trauma » (p. 40), Mesnard, analysant les topoï de mise en exposition, insiste sur les "grandeurs et périls de l'émotion » (p. 64) tout en reconnaissant lui aussi l'apport incontestable de l'expérience artistique au lieu de mémoire.

Car, selon le concept phare de l'œuvre de Gerz analysé par Becker et Debary, c'est bien le «partage du non-vécu » que permet l'œuvre d'art : il s'agit, grâce à l'art, de franchir la distance temporelle et personnelle pour arriver à l'expérience de la violence. «Les chemins ne peuvent qu'être multiples", précise Wahnich, et la réussite de chaque musée ou mémorial se juge, finalement, dans sa capacité à «maintenir des dispositifs de compensation qui savent introduire des écarts là où l'émotion sature l'imagination ", "sans disqualifier le pathos qui est nécessaire à l'intelligence et à la sociabilité » (Mesnard, p. 78).

Grâce à cette analyse de la distance et de l'émotion, l'un des points forts de l'ouvrage est aussi de mettre l'humain au cœur de son objet. Adeline Rispal, architectemuséographe, y est particulièrement sensible dans son analyse de quatre cas sur 
lesquels elle a travaillé. Le point de vue du scénographe, abordant le musée comme «rencontre de l'homme avec l'œuvre de l'homme» (p. 261), apporte un volet très concret à la réflexion. Rispal insiste sur la dimension corporelle de l'expérience du musée de guerre qui confronte le visiteur au corps mutilé, au corps blessé, au corps du soldat reconstitué dans son costume... Cette identification physique aux victimes ou aux acteurs fait de l'expérience du musée de guerre une visite très particulière ; c'est aussi cette dimension humaine qui en fait l'universalité.

11 Enfin, l'ouvrage a le mérite d'aborder, à travers la diversité des auteurs et des cas, un grand nombre de possibilités muséographiques et patrimoniales. L'article de MarcOlivier Gonseth sur l'histoire des expositions du musée de Neuchâtel est particulièrement éclairant. Il y analyse sept modalités d'exposition, qui sont autant de réflexions théoriques et s'articulent autour de trois piliers: montrer, montrer qu'on montre, montrer qu'on ne montre pas. C'est sans doute à cette capacité réflexive, sur des sujets si complexes, que se mesure la qualité d'un projet muséographique. L'analyse que fait Isabelle Rivé-Doré de trois expositions du Centre d'histoire de la Résistance et de la déportation de Lyon, notamment dans leurs différentes utilisations des images, va également dans ce sens, de même que les études de cas européens proposées par Gorgus.

12 «Les violences de guerre peuvent être représentées de très nombreuses manières en fonction des objectifs poursuivis» (p. 257): cette conclusion de Gonseth pourrait s'appliquer à l'ensemble de l'ouvrage. Faisant preuve d'une grande compréhension de son objet, malgré certains oublis inévitables - on regrette notamment le manque d'études des publics, d'études plus approfondies sur les témoins, et l'absence presque complète d'exemples plus lointains, par exemple au Japon, en Afrique ou en Amérique latine -, cet ouvrage apporte une contribution originale et conceptuellement très riche aux études sur la représentation des violences et de l'histoire en général. Il fait de la monstration des violences, dans tous les médias, un élément intrinsèque de la réflexion sur l'histoire et la mémoire en mettant le lien entre les faits d'une part, le spectateur ou visiteur d'autre part, au centre de la réflexion. Au moment où s'ouvre un cycle de commémorations de la première guerre mondiale qui donnera lieu à de nombreuses expositions qui illustreront, n'en doutons pas, bien des aspects analysés ici, cet ouvrage pose un regard original sur des questions très actuelles.

\section{NOTES}

1. Annette Becker est professeur en cultures de la guerre à l'université Paris-Ouest Nanterre La Défense, membre de l'Institut universitaire de France. Elle est notamment l'auteur, avec Stéphane Audoin-Rouzeau, de La Grande Guerre: 1914-1918, Paris, Gallimard, 1998 et de 14-18, retrouver la guerre, Paris, Gallimard (« Bibliothèque des histoires »), 2000.

2. Octave Debary est maître de conférences en anthropologie à l'université Paris-Descartes. Il est spécialiste de la mémoire et des restes, et est notamment l'auteur avec Laurier Turgeon d'Objets, Paris-Québec, Maison des sciences de l'homme et Presses de l'Université Laval, 2007, et avec Mélanie Roustan de Voyage au musée du quai Branly, Paris, La Documentation française, 2012. 
3. Paul Ricœur, La Mémoire, l'Histoire, l'Oubli, Paris, Seuil, 2006, et Temps et Récit III. Le temps raconté, Paris, Seuil, 1985.

4. Voir sur ce sujet : Claire Andrieu, Marie-Claire Lavabre et Danielle Tartakowsky (dir.), Politiques du passé. Usages politiques du passé dans la France contemporaine, Aix-en-Provence, Publications de l'Université de Provence (« Le temps de l'histoire »), 2006.

5. L'exposition a fait l'objet d'une violente polémique, avec des appels à sa fermeture et des menaces à l'encontre de l'équipe du musée en raison de son traitement du «culte des martyrs » de la seconde Intifada en Palestine.

6. L'architecture de Daniel Libeskind est en elle-même porteuse de discours et place le visiteur dans une situation d'identification à l'objet du musée.

\section{AUTEURS}

\section{ANNE-SOLÈNE ROLLAND}

anne-solene.rolland@louvre.fr 